RUNNING HEAD: Build Independent Self-feeding Function: Autism and Feeding Disorders

\title{
Build Independent Self-feeding Function: A Pilot Study on a Child with Autism and Feeding Disorder
}

\author{
Yi Yang ${ }^{1,2}$, Jonathan Tarbox ${ }^{1,3}$, Megan Aclan ${ }^{1}$, Hongen $\mathrm{Ma}^{1}$ \\ ${ }^{1}$ Department of Psychology, University of Southern California \\ ${ }^{2}$ Department of Psychology, University of Edinburgh \\ ${ }^{3}$ FirstSteps for Kids
}

Yi Yang: s2060087@ed.ac.uk

Jonathan Tarbox: jtarbox@usc.edu

Megan Aclan: aclan@usc.edu

Hongen Ma: hongenma@usc.edu

*Correspondent author at: Department of Psychology, University of Southern California, SGM

501, Los Angeles, CA 90089, USA; email: jtarbox@usc.edu 


\begin{abstract}
The high occurrence of feeding disorders among children with autism spectrum disorder has caught the attention of professionals from different disciplines. When taking an insight into their feeding problems, mixed physical, behavioral and psychological difficulties were encountered, such as oral skill deficits (e.g., chew, swallow, and coordination), fine motor skill deficits, food selectivity (e.g., texture aversion), food neophobia, and so on. However, the traditional non-oral tube feeding method for maintaining their nutrition consumption is criticized as too intrusive and inadequate for training self-feeding skills. Therefore, to target both establishing independent selffeeding function and food consumption variety (nutrition) while using less intrusive intervention method, the present study tested the effectiveness of a multicomponent behavioral intervention protocol on a child with autism and feeding disorder, setting across physical, behavioral and psychological background. A task analysis on self-feeding function resulted in a backward-chain training procedure on swallowing, chewing, and fine motor skills, separately and then combined. The training procedure consisted of 3 key components: 1) swallowing training, 2) chewing training starting with using a chew facilitator tube, 3) fine motor training targeting on self-feeding; and 4) fine motor-chewing-swallowing coordination training with multiple types of food. The results showed the present multicomponent behavioral intervention protocol successfully established the participant's self-feeding skills: ameliorated his oral and fine motor skills, cultivated his independent self-feeding function, and increased his food consumption volume and variety.
\end{abstract}

Keywords: Autism; ASD; Feeding Disorder; Applied Behavior Analysis; Oral-Delay; Fine Motor Skill; Changing Criteria Design; Task Analysis. 


\section{Introduction}

As an increasingly common neurodevelopmental disorder, Autism spectrum disorder (ASD) was increasing its prevalence, for example, surging from 1 in 150 in 2000 to 1 in 54 in 2016 in the USA, according to the Center for Disease Control and Prevention of the USA (Maenner et al., 2020). ASD is mainly charactered by social skill deficits, repetitive and stereotyped behaviors, and impaired and restricted communication (5th ed.; DSM-5; American Psychiatric Association, 2013). ASD is often comorbid with feeding disorders. It was recorded that up to $89 \%$ of children with ASD exhibited some forms of feeding difficulties varying by behavioral topography, such as food neophobia, food refusal, rigidity with food types and textures, rigid routines around mealtime, and other challenging disruptive behaviors when non-preferred food was presented (Ahearn, Castine, Nault, \& Green, 2001; Bandini et al., 2010; Bowers, 2002; Field , Garland, \& Williams, 2003; Ledford \& Gast, 2006; Marshall , Hill, Ziviani, \& Dodrill, 2014; Schmitt et al., 2008; Schreck, Williams, \& Smith, 2004; Tarbox \& Burmudez, 2017; Tarbox, Schiff, \& Najdowski, 2010). In the study of Mayes and Zickgraf (2019) on 1462 youth, 70.4\% of children with autism showed atypical eating behaviors, comparing to $4.8 \%$ of the general population. Besides, among all the feeding disorder groups, the ASD group has less nutritional variety (Berlin et al., 2011; Schreck et al., 2004). Therefore, it is of significance to address the feeding problem among ASD patients, as well as to take a thorough understanding of feeding disorders before developing an intervention program for them.

Feeding disorder is an umbrella term. The World Health Organization International Classification of Functioning, Disability, and Health has proposed a definition for the term "pediatric feeding disorder" as the impairment or age-inappropriate oral intake, including medical (e.g., aspiration, dysphagia), nutritional (e.g., concomitant malnutrition), feeding skills (e.g., fine 
motor, food selectivity), and/or psychosocial dysfunction (e.g., disruptive behaviors) (Goday et al., 2019; Kovacic et al., 2021; World Health Organization, 2001). According to how nutrition is received, pediatric feeding disorders can be classified as tube dependent (non-oral nutrition consumption), liquid dependent (consume liquids orally), and food selectivity (consume orally with food restrictions), which also reflected the severity of the problem (Greer, Gulotta, Masler, \& Laud, 2008). The enteral/non-oral tube feeding was deemed as the severest form and had received much criticism (Morris, 1989; Taylor, Kozlowski, \& Girolami, 2017; Williams, Riegel, Gibbons \& Field, 2007). Despite non-oral tube feeding is common in medical treatment to deal with oral deficits and is adequate to increase nutrition intake with immediate effectiveness, it could neither help to establish adaptive independent self-feeding function, nor to treat feeding disorder itself (Taylor, Kozlowski, \& Girolami, 2017). The complicated problem of tube feeding is that once it started, the oral sensory perception of the child would decrease, and his/her mouth later would become hypersensitive and hyperirritable while the oral-motor skills would degenerate if oral-feeding has already started, which further makes the oral intake experience more aversive for the child (Morris, 1989). Besides, the enteral tube feeding is frequently viewed by parents as a symbol of severe damage and a failure of nurturing, which is more likely to stress them to skip the preliminary steps that were necessary for developing oral-motor competency. It is also worth noting that parenting stress may lead to forced feeding of the feeders as well, which in return brings feeding aversion of the child with oral deficits and cause the child to develop disruptive behaviors to cope with aversive feeding situations (Babbitt et al., 1994; Goday et al., 2019). Therefore, from non-oral tube feeding to disruptive feeding behaviors, feeding problems were shaped by mixed physical, behavioral, and psychological problems, suggesting it is necessary to take a multidisciplinary consideration when developing an intervention program. 
Given the above-mentioned situations, it is of long-term benefit to train independent selffeeding skills of those individuals who exhibited the deficits, and more ethical to use less intrusive or traumatizing intervention methods for building adaptive oral intake function while addressing the nutrition consequence. Multidiscipline based behavioral intervention is an optimized choice to address these purposes. On the one hand, it was well-evidenced by plenty of previous studies regarding the effectiveness of behavioral treatment on food selectivity and rigidity of children with autism by promoting adaptive while extinguishing disruptive behaviors (e.g., Ledford \& Gast, 2006; Seiverling, Hendy, \& Williams, 2011; Sharp, Jaquess, Morton, \& Herzinger, 2010). A review of 16 studies, addressing the eating habits of 18 children with ASD, identified the successful behavioral intervention strategies, including differential reinforcement contingent upon appropriate eating behaviors, simultaneous and/or sequential presentation of preferred and nonpreferred food, stimulus fading, escape extinction, and so on (Ledford \& Gast, 2006). Laud and colleagues (2009) directly observed the treatment outcomes for a variety of feeding problems among 46 children with ASD and substantiated the effectiveness of intensive ABA treatment. Its follow-up data also showed positive results regarding consumed food variety, texture advancement, protocol usage, and caregiver recommendation. On the other hand, beside its effectiveness on the relatively mild type of feeding problems ( e.g., food selectivity; Tarbox \& Burmudez, 2010), behavioral treatment was also evidenced to build oral intake function among those with severe intake deficits. A randomized controlled group study on 4-36 months non-oral tube fed infants indicated that behavioral intervention successfully eliminated the tube feeding for $47 \%$ of the behavioral intervention group, contrasting to none of the nutrition-alone intervention group was succussed (Benoit, Wang, \& Zlotkin, 2000). Another clinical study, using intensive behavioral therapy to treat children who were dependent on supplemental tube feedings, found 31 out of 46 
children were successfully weaned from their feeding tube while 14 children reduced $50 \%$ or more of tube feeding (Williams et al., 2007). The study of Taylor and colleagues (2017) also confirmed the success of behavior intervention in the cases in which medical or oral motor factors were involved. All these findings substantiated the efficacy of behavioral therapy, while demonstrated it was a more cost-effective while less intrusive method comparing to the non-oral tube feedings.

In fact, a systematic review and meta-analysis study on feeding disorder treatments by Sharp and colleagues ( Sharp, Volkert, Scahill, McCracken, \& McElhanon, 2017) acknowledged the central role of behavioral interventions on increasing oral intake of children with feeding disorders, and recommended multidisciplinary assessment and care. There are also a few studies that developed successful behavioral protocols to increase chewing behavior on a variety of food types for children with feeding disorders who exhibited inadequate chews per bite (Volkert, Piazza, Vaz, \& Frese, 2013; Volkert, Peterson, Zeleny, \& Piazza, 2014). However, relatively less behavioral intervention research addressed the issue of oral motor deficits, and even fewer studies focused on establishing a comprehensive independent self-feeding function covering oral motor (e.g., suck, swallow, chew and coordination), fine motor (eyes, shoulders, arms, and hands and coordination), and food rigidity issues among children with autism and feeding disorders. The systematic review of Sharp and colleagues (2017) also highlighted the shortage of experts as well as a uniform treatment framework in this field. Given the central role that behavioral intervention plays in dealing with autism and feeding disorder, it is feasible to develop a long-term effectiveness focused, and less intrusive intervention protocol for behavior analysts as a practice reference when encountering oral motor deficits, since most of the intervention load has been placed on in-home services in reality. Unquestionably, behavioral analysts are necessarily to cooperate with experts specialized in medicine, nutrition and psychotherapy, given the complicity of oral intake function. 
However, applied behavior analysis plays a central role in treating feeding disorders. Its techniques, including functional analysis, motivating and reinforcement operations, task analysis, chaining, and so on (Sharp et al., 2017), are effective to break down the complicated feeding coordination into adaptive, acceptable, and adequately small steps for patients to practice step by step, safely and confidently. In conclusion, establishing the independent self-feeding function, including training motor skills, is within the competency scope of behavioral treatment.

\section{Present Study}

With addressing physical, psychological and nutritional factors, the present study aimed at testing the effectiveness of a behavioral intervention protocol using behavioral techniques, including task analysis (addressing physical deficits), chaining, stimulus fading (addressing fearful reactions and physical deficits), positive reinforcement with allowing escape (addressing psychological stress), negative reinforcement, and a changing criteria design, to train a child with autism to establish independent self-feeding function while increasing the variety of food he accepted for consumption.

\section{Methods}

\subsection{Participant and Setting}

Allen was a 5-year-old boy with autism and feeding disorders who is in good health but thin when starting the session. He was also diagnosed with developmental delay and had started to receive ABA services at age 4 and speech therapy at age 3 at school. His mother reported he was an infant born premature, and the previous pediatric diagnosis documents also assessed Allen as "failure to thrive and mild microcephalus". Delayed fine motor skills of Allen were also confirmed. Until before the COVID-19 pandemic, Allen was receiving 10 hours per week of homebased behavioral intervention, which was designed to address his language, social and motor 
deficits. After in-home services were disrupted by the COVID-19 pandemic, Allen received most sessions virtually during the pandemic, with the intervention operations implemented via Telehealth with the help of his mother. Since his mother had received regular parent training before the pandemic in how to implement behavioral techniques on Allen during daily life, she was qualified to help performing the feeding intervention. At the beginning of the training, Allen could follow simple imitation directions, such as "touch your nose", "clap your hands", "give me..." and so on. Allen had limited language skills comparing to the typical children of the same age. However, he could use "yes" or "no" to express his preferences when was asked about the activities or toys to engage, and could echoic when teaching him new objects. He also could mand for preferred objects with saying the name of the object, such as "London Bridge" for his favorite music, and he was leaning to use "I want..." to mand for preferred things.

Allen was assessed as feeding disorder before the ABA treatment. He refused to try other food textures except for fruits purified by a blender. He also refused to be fed by the teachers at school for lunch, and only received breakfast and dinner at home. Despite Allen exhibited chewing behaviors on toys and plastic things using teeth and moving those objects inside his mouth using tongue for oral sensory, he did not show any chewing behavior on food at all. Before the start of intervention, When his mother introduced small chopped soft fruits, Allen smashed them with his tongue against palate without using his teeth. Although Allen could swallow liquids, such as water and juice, he was not capable of swallowing down the age-appropriate size of purified fruits without the facilitation of water or juice. If without injecting a sip of water or juice using a syringe into his mouth immediately after he received a spoon of food, he would gagge and spit out all the food, or sometimes vomit. When Allen was asked to pick up the spoon in the bowl with food presence, he would escape. However, when he was required to scoop and transport beans from a 
container to another, he could not do it without spilling out. Therefore, evidenced by the baseline observation and his mother's self-reporting, except extreme aversion and avoidance behaviors towards food consumption, Allen showed limited swallowing skill, absent food chewing behavior despite capable of chewing toys, and poor fine motor skill for independent self-feeding.

In the present study, a self-feeding task was defined as a chain of independent behaviors: using utensils to transport food from a container to mouth, chewing the food with teeth while stirring with tongue, and swallowing down the masticated food, which mainly involved 3 parts of different skills (fine motor skill, chewing, and swallowing) and their coordination. In addition, according to the fatalness of those skills, the present study separated the whole independent selffeeding task into 3 backward-chain training steps: swallowing training, chewing training, and hand fine motor training. The targets of the present protocol include: 1. train Allen to chew ageappropriate chunk food with teeth; 2. train Allen to consume food of different textures, including

fruits, rice, noodle, snacks, vegetables, and meats; 3. train Allen to master independent self-feeding fine motor skills.

All sessions were conducted in a separated playing room in his house, with a child-sized table and a chair prepared. Materials included food, a food container, a spoon, a plastic chew facilitator tube, and his preferred objects toys, such as tablet with his favorite videos and games, a spray bottle with water, and a phone with his favorite classical music. In addition, an iPhone was utilized for video-taping the sessions for later scoring interobserver agreement (IOA).

\subsection{Experimental Design and Measurement}

A $10 \mathrm{~cm}$-long $1 \mathrm{~mm}$-thick and $1 \mathrm{~cm}$-diameter transparent, flexible plastic soft tube was tailored as the tool instead of spoon to train him chewing food at the beginning. Along one end of the tube, a $2 \mathrm{~cm}$-long opening was cut with a smooth angle for holding food inside for chewing 
(Volkert et al., 2014). The shape of the cut opening could contain solid food tightly to prevent it from moving around in the mouth, therefore, prevent Allen from choking. By holding the other end of the tube facilitator, the feeder could also make sure that each chew the participant made was right on the tube facilitator with food, despite the details in the mouth could not be seen during chewing. Other materials included feeding spoons and forks, food container, a student chair and table, gloves, tablet, and other reinforcers.

Table 1. Oral motor Skill Training

\begin{tabular}{|l|l|l|}
\hline \multicolumn{1}{|c|}{ Oral motor } & \multicolumn{1}{|c|}{ Skill Training } & \multicolumn{1}{c|}{ Behavioral Technique } \\
\hline Swallow & $\begin{array}{l}\text { Subtly increase the density/size of the } \\
\text { puree/smashed food until reaching a rice size }\end{array}$ & $\begin{array}{l}\text { Stimulus fading; } \\
\text { Non-removal spoon; } \\
\text { Negative reinforcement }\end{array}$ \\
\hline Chew & $\begin{array}{l}\text { Subtly increase solid food size from a rice size; } \\
\text { From not require swallowing to swallowing }\end{array}$ & $\begin{array}{l}\text { Stimulus fading; } \\
\text { Positive reinforcement allowing } \\
\text { escape }\end{array}$ \\
\hline $\begin{array}{l}\text { Chew-Swallow } \\
\text { Coordination }\end{array}$ & $\begin{array}{l}\text { From using smashed food, to dissolvable solid } \\
\text { puffs, to soft solid food, and to hard solid food }\end{array}$ & $\begin{array}{l}\text { Chaining; } \\
\text { positive/negative reinforcement }\end{array}$ \\
\hline
\end{tabular}

Table 2. Fine motor Skill Training

\begin{tabular}{|c|c|c|}
\hline Fine motor & Skill Training & Behavioral Technique \\
\hline Scoop & $\begin{array}{l}\text { Scoop beans from a bowl and stably stay in the } \\
\text { air for 3-5 seconds without dropping beans }\end{array}$ & \multirow{3}{*}{$\begin{array}{l}\text { Chaining; } \\
\text { Prompt; } \\
\text { Positive reinforcement }\end{array}$} \\
\hline Transport & $\begin{array}{l}\text { Scoop and transport beans from one bowl to } \\
\text { another bowl }\end{array}$ & \\
\hline $\begin{array}{l}\text { Scop-transport } \\
\text { Coordination }\end{array}$ & $\begin{array}{l}\text { Scoop puree/smashed food from a bowl to } \\
\text { mouth with prompt fading }\end{array}$ & \\
\hline
\end{tabular}

A task analysis based on Allen's specific condition was conducted. According to it, the whole intervention procedure was consisted of: 1)baseline, 2)swallowing training using puree food, 3)swallowing training as in stage 2 plus chewing training using tube facilitator, 4) chewing training 
as in stage 3 plus fine motor-swallow coordination training, 5) fine motor-swallow coordination training as in stage 4 plus chew-swallow coordination training, 6) fine motor-chew-swallow coordination training. The number of chews per bite of food, volume of food for self-feeding, and percent of self-feeding trials per session were recorded for data analysis. A changing criteria design was adopted to control and evaluate training effectiveness. The standard for chews per bite of food was increasing (occasionally decreased to check the experimental control) along with the progress of sessions, for example, changing from 5 chews to 10 chews, to 15 chews as participant achieved the previous standard. The operational definition for a successful trial was: the number of chews on the tube facilitator or food met the criteria of chews for the current trial.

\subsection{Procedure}

\subsubsection{Stage 1: Baseline}

A functional analysis was conducted to evaluate Allen's before intervention feeding state. During baseline phase, 1/3 bowl of smashed fruits and a spoon of age-appropriate size was presented to Allen. An instruction, “Allen, take a bite. If you feel uncomfortable, you can spit out”, was given by researcher or his mother to Allen. Three trials per feeder were performed on Allen, resulting in him escaping from the session room and showing disruptive behaviors without touching the spoon. Once the bowl of food was removed from the feeding table or he was told that he would not be fed, he stopped his disruptive behaviors and came back to the session room. To show the researcher how he was fed in daily time, his mother ended up having to grasp one of his wrists to prevent him from escaping, and only let him go after he received and swallowed down one spoon of food. This whole process was along with Allen's crying, showing aversive facial expression, gagging, and spitting out the food for 2 trials and vomiting for 1 trial when him trying to swallow. He also kept packing food in his mouth and could only swallow it down after receiving 
a syringe of juice injected into his mouth by his mother, with no food chewing behaviors exhibited.

Therefore, his daily feeding process was necessary to pair a spoon of food with an injection of juice/water, signaling no chewing skill, limited swallowing skill, and chew-swallow coordination deficit.

Table 3. Shaping and Chaining Procedure for Independent Self-feeding

\begin{tabular}{|c|c|c|c|c|c|}
\hline \multicolumn{6}{|c|}{ Self-feeding process } \\
\hline \multirow{2}{*}{ 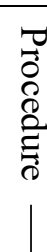 } & Stage Goal & Fine motor & Chew & Swallow & Behavioral Technique \\
\hline & S1: Baseline & Limited & No skill & Limited & Functional analysis \\
\hline $\begin{array}{l}\text { E } \\
1\end{array}$ & S2: Swallow & \multirow[b]{2}{*}{ N/A } & N/A & Puree food & $\begin{array}{l}\text { Stimulus fading for food } \\
\text { texture; } \\
\text { non-removal Spoon; } \\
\text { negative reinforcement for } \\
\text { swallowing }\end{array}$ \\
\hline & $\begin{array}{l}\text { S3: } \\
\text { Swallow; } \\
\text { Chew }\end{array}$ & & \multirow{2}{*}{$\begin{array}{l}\text { Smashed/small } \\
\text { size food } \\
\text { facilitated by a } \\
\text { tube facilitator }\end{array}$} & \multirow{2}{*}{$\begin{array}{l}\text { Smashed } \\
\text { food }\end{array}$} & $\begin{array}{l}\text { Stimulus fading for food } \\
\text { texture; } \\
\text { negative reinforcement for } \\
\text { swallowing; } \\
\text { positive reinforcement } \\
\text { allowing escape for } \\
\text { chewing }\end{array}$ \\
\hline & $\begin{array}{l}\text { S4: } \\
\text { Hand-Swallow } \\
\text { Coordination; } \\
\text { Chew }\end{array}$ & \multirow[t]{2}{*}{$\begin{array}{l}\text { Scoop and } \\
\text { self-feed } \\
\text { puree/ } \\
\text { smashed food }\end{array}$} & & & $\begin{array}{l}\text { Chaining for coordination; } \\
\text { Prompt for coordination; } \\
\text { negative reinforcement for } \\
\text { coordination; } \\
\text { positive reinforcement } \\
\text { allowing escape for } \\
\text { chewing }\end{array}$ \\
\hline$\downarrow$ & $\begin{array}{l}\text { S5: } \\
\text { Chew-Swallow } \\
\text { Coordination }\end{array}$ & & \multicolumn{2}{|c|}{$\begin{array}{l}\text { Chew smashed/chopped and } \\
\text { small solid food with variation }\end{array}$} & $\begin{array}{l}\text { Chaining for coordination; } \\
\text { Prompt for coordination; }\end{array}$ \\
\hline
\end{tabular}




\begin{tabular}{|c|c|c|}
\hline $\begin{array}{l}\text { S6: Hand- } \\
\text { Chew-Swallow } \\
\text { Coordination }\end{array}$ & $\begin{array}{l}\text { Self-feed with little prompt, chew and swallow } \\
\text { solid food with variation }\end{array}$ & $\begin{array}{l}\text { Positive/negative } \\
\text { reinforcement for } \\
\text { coordination }\end{array}$ \\
\hline
\end{tabular}

\subsubsection{Stage 2: Swallowing Training with Puree Food}

After taking the baseline data and before starting the chewing training, the swallowing training was taken place first with using puree food, which helped Allen to gradually accept the new feeding procedure performed by researcher or his mother. While fading out the syringe injection of juice, a special designed trainer cup, which could prevent water from pouring on the face during drinking, was employed to train Allen with independent water consumption skill to facilitate himself swallowing down food.

At the beginning of the first few sessions, Allen's mother was present to feed the first several spoons of food, and then transfer the rest of the feeding procedure to the researcher while she stayed aside. Gradually, his mother faded out from the feeding process until finally out the session room in the following sessions. There was no physical restriction involved when researcher fed Allen. Instead, researcher used negative reinforcement procedure to promote Allen's consumption and cooperation behaviors, for example, removing the tablet which playing his favorite videos. Likewise, the injection procedure by his mother was gradually faded out by letting himself drink water to help swallowing using a trainer cup instead. And the researcher would prompt Allen to take one sip of drink in the trainer cup after he was fed one spoon of food by the researcher. Once Allen could accept the food without gagging or vomiting, two spoons of food pairing one sip of drink was implemented, and so on. Then gradually, he was trained to drink water on his own initiative, by picking up the cup, taking a sip of drink and putting the cup back on the 
table independently. When Allen could swallow down puree food smoothly for above 95\% trials for 3 consecutive sessions, the next stage of intervention was introduced.

During the sessions, a tablet playing his favorite videos/games was used as reinforcement all through the feeding procedure. A few backup reinforcers he adored were also prepared, such as bubble and water spray. Once he refused to take a spoon of food, researcher would remove the reinforcer, by stopping the video/game, or using hand to block his view from the tablet, or block his hand from touching the tablet to continue, or remove the tablet, until he finished swallowing down the spoon of food.

\subsubsection{Stage 3: Swallowing Training as Stage 2 Plus Chewing Training with Tube}

\section{facilitator}

Chewing training was carried out independent of the swallowing procedure for both ability and metal stress consideration. As the swallowing training in stage 2 still going on separately, with increasing the food texture gradually until the smashed particles as big as rice size (stimulus fading), a chewing training procedure was introduced with using a tube facilitator to ensure his safety and learning success.

Because Allen was very vigilant toward anything that others tried to bring approaching his mouth area, a stimulus fading procedure (feared stimuli gradually approaches participant, allowing time for him to habituate; Furr, Sanchez, Hong, \& Comer, 2020) regarding the tube facilitator was performed to desensitize Allen's vigilant reaction, and let him gradually accepting feeders to put the tube facilitator with food into his mouth. The procedure began with showing the tube for one instant (present the tube facilitator in front of him) with $60 \mathrm{~cm}$ away from Allen, then gradually shortening the distance between the tube and Allen and finally touching his lips, while increasing the presence timing of the tube from one instant to 3 seconds. Three trials were performed for each 
stimulus fading step. After Allen allowing the tube facilitator touching his lips for 3 consecutive trials, researcher then began to move the tube in his mouth little by little forward and ended at reaching his back-teeth part with giving "open mouth" instruction.

Before each session, the reinforcers (tablet, spray bottle, babble, umbrella) were withheld by his parents for at least 4 hours to ensure their effectiveness. Three trials per hour during session were performed, with 1 data point on the changing criteria graph representing the average number of chews per trial within 1 session. During each trial, researcher put the tube facilitator at either side of Allen's back-teeth, instructing "chew the tube", while modeling the chewing behavior for Allen. Once Allen chewed at the tip part of the tube facilitator, research counted out loudly the number of chews Allen made, prompting him to finish the required number of chews by the criteria. The criteria of chews for the next session was set according to the performance of the previous session. When Allen did not meet the criteria, he was encouraged to chew more with receiving the instruction "Let's chew X more times". When he met the criteria for chews, researcher withdrew the tube facilitator immediately and gave Allen his preferred reinforcer, such as a tablet or water spray bottle. As session went on, the food varied from smashed fruits to the mixture of soft rice with chopped/smashed vegetables and meat in appropriate tiny size for him to swallow. After he accepted chewing the tube facilitator held by researcher for 3 consecutive trials with meeting the criteria for 3 consecutive sessions, researcher started to let Allen pick up the tube facilitator with food, put into mouth and place at the back teeth part to chew all by himself. After chewing the tube, swallowing was not required during this stage.

Beside oral motor skill training, food selectivity problem was also addressed at the same time. For expanding his food consumption variety, we started with rotating different types of baby food (e.g., fruit, meat, vegetables) for chewing, which were sold in supermarket. After he accepted 
the texture of the baby food, smashed food of slightly bigger particles made by a blender was adopted as the chewing material. Then after he accepted to chew the smashed food, solid puffs of various shapes and tastes that could be melted by saliva (reduce the risk of choking) were used for chewing by placing in the tube facilitator. After he accepted to chew on every trial of puff using the tube facilitator, the tube facilitator was fade out and puffs were given directly to his hand to put into mouth by himself. It is worth noting that at this stage of chewing training, Allen was not required to swallow down the food he chewed, despite the chewed food would naturally be consumed by him to some degree.

\subsubsection{Stage 4: Chewing Training as Stage 3 Plus Fine Motor-Swallow Coordination}

While the chewing training in stage 3 still going on to increase food consumption variety, the fine motor and swallowing coordination training was introduced to train Allen to feed himself under the researcher's physical prompt.

With receiving most-to-least physical prompt from the researcher, Allen began fine motor training with scooping beans from one container staying for 3-5 seconds in the air and then put back in the container. After mastering that, he was trained to scoop and move a spoon of beans stably from a container to drop in another. At the same time, other fine motor skills were also carried out targeting on improving his finger and wrist flexibility and stability by using behavior imitation procedure (e.g., say "do this" to ask him to imitate waving his wrist towards himself while holding a spoon in the same way with self-feeding). After Allen's success for above $85 \%$ of the trails scooping beans for 3 consecutive session without prompting, the researcher began to train him to scoop and transport smashed food steadily from container into his mouth for swallowing. After he could bring a spoon of smashed food to his mouth by himself with no more than $1 / 5$ of 
food spilling for above $85 \%$ trials for 3 consecutive sessions, the fine motor-swallow coordination training moved to stage 6 .

\subsubsection{Stage 5: Fine Motor-Swallow Coordination as Stage 4, Plus Chew-Swallow}

\section{Coordination}

While the fine motor-swallowing coordination training in stage 4 was still going on, and Allen already accepted to chew a variety of smashed food/puffs in his mouth, researcher began to positively reinforce Allen to swallow down the food he had chewed during the chewing training.

The goal during this stage was to gradually pair spoon feeding with chewing various solid food and with swallowing down. The dissolvable puffs were used before other solid food (e.g., fruit, noodle, meat) to reduce the danger of getting choked if chew-swallow coordination failed, then gradually other daily solid food was introduced after his chewing skill on puffs met the criteria. The tube facilitator was also faded out when Allen became more and more comfortable with receiving food directly, as well as chew-swallow coordinated smoothly. Researcher started with only giving the puff at the beginning of this stage, then gradually added new types of solid food for chewing (round shape yogurt taste puff, cheese ball, Kix, Granola, various shapes of cheerios, and dry cranberry). During each session, the different types of food were placed in a bowl, and Allen was required to chew for 10-15 times or more on each bite of food according to the changing criteria as well as the hardness of the food. Allen only received reinforcers after finishing the number of chews that met the criteria. After he was capable of chewing more than 10 types of solid food with meeting the criteria for above $85 \%$ trials for 3 consecutive sessions, the stage 6 of intervention was introduced.

\subsubsection{Stage 6: Fine Motor-Chew-Swallow Chain Coordination}


At the beginning of stage 6 , the fine motor-swallowing (smashed food) procedure and chewing-swallowing (solid food) procedure were paired and took place intermittently during mealtime. For example, researcher started with letting Allen swallow down 3 spoons of smashed food, then let him chew 1 piece of solid food (e.g., rice, noodle, meat, bean) for more than 15-25 times according to criteria and swallow down. Gradually, researcher decreased the frequency of smashed food feeding and increased the frequency of solid food feeding, resulting in Allen's consumption amount of smashed food decreased while the amount of solid food consumption increased. Researcher also started to training Allen using spoon/fork to transport solid food to his mouth, then to chew and swallow down, using most-to-least prompt. After Allen was able to take $70 \%$ of his meal with solid food and $30 \%$ with smashed food for 3 consecutive sessions, stage 6 finished, and parent training while skill maintenance stage began.

Follow-up data showed that the family was able to maintain the improvements on independent self-feeding behaviors of Allen more than 3 months after this training intervention ended.

\subsection{Response Measurement and Interobserver Agreement}

The sessions were videotaped with an iPhone camera by the researcher. A secondary observer watched the videos and collected data using identical measurement procedures as the primary data collector. Interobserver agreement on evaluating the data were calculated for $90 \%$ of the sessions distributed evenly across baseline, intervention, and follow-up phases. Total count interobserver agreement was calculated for the number of chews and the percent of independent self-feeding trials, resulting in 91.8 and $93.3 \%$, respectively.

\section{Results and Discussion}




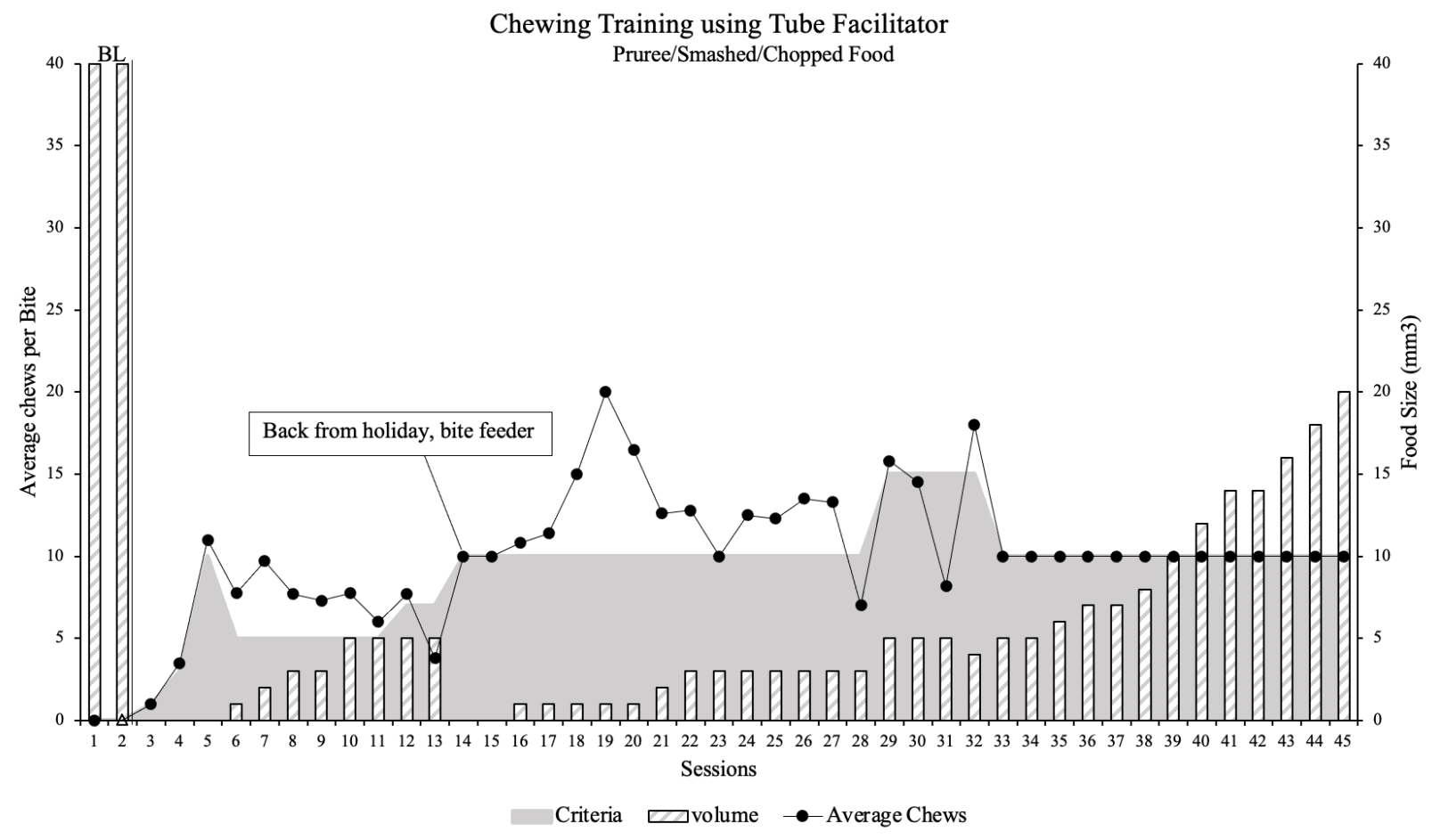

Figure 1. Average chews per bite of food with changing criteria design

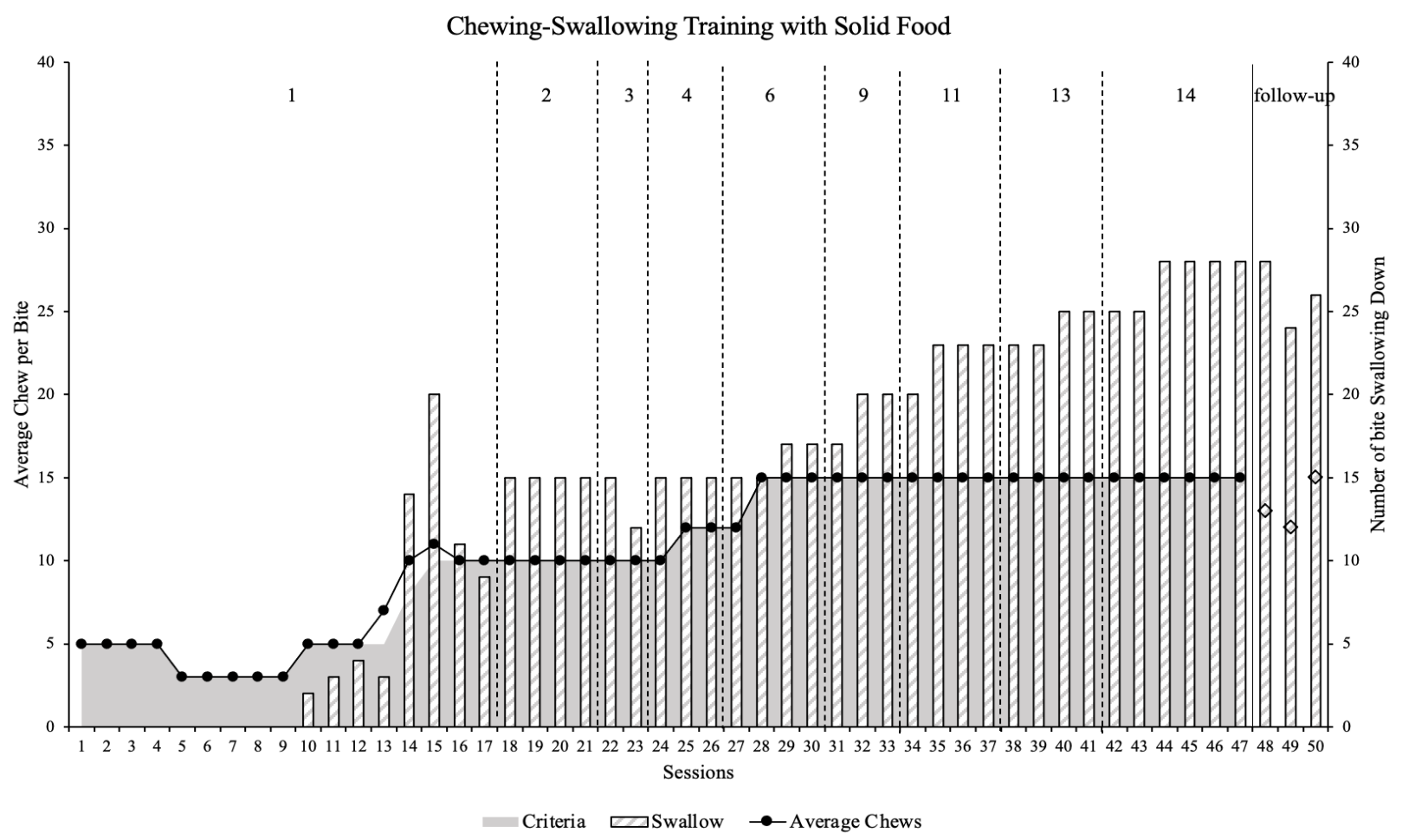

Figure 2. Average chews per bite of food with changing criteria design

(Numbers on top of the graph represent how many types of food used during each phase of sessions.) 


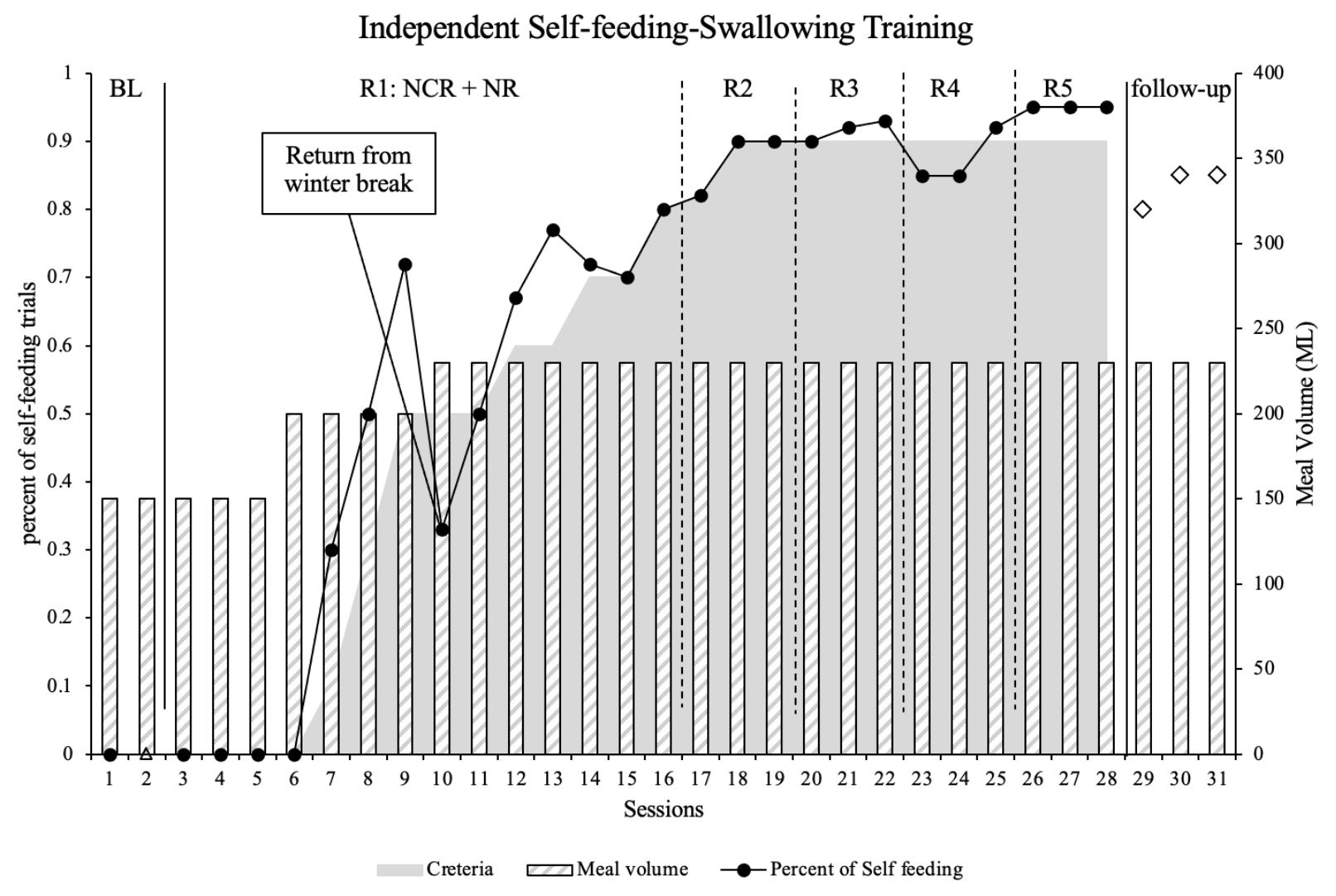

Figure 3. Average chews per bite of food with changing criteria design ( $\mathrm{R}=$ reinforcement ratio, e.g., $\mathrm{R} 3=$ access to preferred item after taking in 3 spoons of food.)

As demonstrated in Figure 1, 2, and 3, the self-feeding protocol in the present study successfully ameliorated Allen's oral motor deficit, established his fine motor-chew-swallow coordination, built his independent self-feeding function, and increased both his food consumption volume and variety. During baseline, Allen showed limited swallowing function, no chewing function, low fine motor skills for self-feeding, and no self-feeding coordination at all. After receiving 13 months onsite and online sessions based on the present protocol, Allen could chew food with age-appropriate size, as well as consume a variety of food types ranging from fruits to rice, noodles, vegetables, and meats, despite slower than his 1 year younger typical sisters. 
It was worth noting that Allen exhibited great interested to scoop food and use spoon/fork by himself once he was taught to masker the skills, and enjoyed doing them on his own and learned faster than these procedures operated by a feeder. Besides, after he could smoothly take in and swallowing down the smashed food, one session he said "I want eat" to the researcher with looking into researcher's eyes when researcher arrived at his home. Therefore, it seems after the participant learned how to control the self-feeding procedure previously operated by others, his fearful and aversive responses also decreased quickly.

There are a few limitations of this intervention case study in addressing feeding disorders with oral deficits. First, the present protocol was performed on a participant with autism and oralmotor deficits, who was already capable of sucking and swallowing liquid before the intervention. Therefore, the swallowing training was relatively not very tough to address in this study. However, many feeding disorders involve more fundamental and severe oral motor deficits, such as dysphagia and other swallowing coordination deficits. More subtle and basic training is required in that case. For example, therapists have to training the tone and movement of jaw (critical for rhythmic movements), lips and cheeks (benefit swallowing preparation), and tongue (pass the bolus from front to back within the mouth) when necessary (Morris, 1989). Second, the present protocol did not include a program for rhythmicity training, which was critical for the whole feeding coordination, especially for oral deficits such as sucking, for choking prevention. Despite the researcher did counting as rhythming for the chewing and chew-swallow coordination training, the protocol did not emphasize the rhythmicity training enough, as the participant did not suffer from the safety concern of choking from abnormal swallowing or reflux problem. However, plenty of research on ASD has found that autistic individuals have problem with timing, which plays a central role in rhythmicity, and could further influence the coordination of jaw, lips, and tongue 
movements. A recent systematic review study evidenced that autistic participants showed reduced temporal synchrony tendencies in audio-visual, audio-motor, visuo-tactile, visuo-motor, social motor, and conversational sensory processing across 32 studies (Murat Baldwin, Xiao, \& Murray, 2021). Another genetic study also confirmed the timing deficit of autism by revealing ASDassociated changes in the maturational sequence of early neuron development, involving temporal dysregulation of specific gene networks and morphological growth acceleration (Schafer et al., 2019). In addition, the present study was a single case study, it is necessary to replicate the training protocol on more children who have autism and/or feeding disorders in the future.

In conclusion, the present protocol operationalized the complex self-feeding skills into separate small steps (swallow, chew, scoop, self-feed) using task analysis, and trained participant step-by-step based on the ABA techniques. These operations were effective in promoting the child with autism and feeding disorders to master independent self-feeding skills in his own pace. Since plenty of studies provide firm evidence that children with ASD display atypical feeding patterns which place them at risk for significant nutritional deficits, it is of great social significance for the present study to explore effective while less intrusive interventions for the feeding problems of children with autism. 


\section{References}

Ahearn, W. H., Castine, T., Nault, K., \& Green, G. (2001). An Assessment of Food Acceptance in Children with Autism or Pervasive Developmental Disorder-Not Otherwise Specified. Journal of Autism and Developmental Disorders, 31(5), 505-511. https://doi.org/10.1023/A:1012221026124

American Psychiatric Association, \& American Psychiatric Association (Eds.). (2013).

Diagnostic and statistical manual of mental disorders: DSM-5 (5th ed). American

Psychiatric Association.

Babbitt, R. L., Hoch, T. A., Coe, D., Cataldo, M. F., Kelly, K. J., Stackhouse, C., \& Perman, J. A. (1994). Behavioral assessment and treatment of pediatric feeding disorders. Journal of Developmental and Behavioral Pediatrics, 15(4), 278-291. https://doi.org/10.1097/00004703-199408000-00011

Bandini, L. G., Anderson, S. E., Curtin, C., Cermak, S., Evans, E. W., Scampini, R., Maslin, M., \& Must, A. (2010). Food Selectivity in Children with Autism Spectrum Disorders and Typically Developing Children. The Journal of Pediatrics, 157(2), 259-264. https://doi.org/10.1016/j.jpeds.2010.02.013

Benoit, D., Wang, E. E. L., \& Zlotkin, S. H. (2000). Discontinuation of enterostomy tube feeding by behavioral treatment in early childhood: A randomized controlled trial. The Journal of Pediatrics, 137(4), 498-503. https://doi.org/10.1067/mpd.2000.108397

Berlin, K. S., Lobato, D. J., Pinkos, B., Cerezo, C. S., \& LeLeiko, N. S. (2011). Patterns of Medical and Developmental Comorbidities Among Children Presenting With Feeding Problems: A Latent Class Analysis. Journal of Developmental \& Behavioral Pediatrics, 32(1), 41-47. https://doi.org/10.1097/DBP.0b013e318203e06d 
Bowers, L. (2002). An audit of referrals of children with autistic spectrum disorder to the dietetic service. Journal of Human Nutrition and Dietetics, 15(2), 141-144.

https://doi.org/10.1046/j.1365-277X.2002.00345.x

Field, D., Garland, M., \& Williams, K. (2003). Correlates of specific childhood feeding problems. Journal of Paediatrics and Child Health, 39(4), 299-304. https://doi.org/10.1046/j.1440-1754.2003.00151.x

Furr, J. M., Sanchez, A. L., Hong, N., \& Comer, J. S. (2020). Exposure therapy for childhood selective mutism: Principles, practices, and procedures. In Exposure Therapy for Children with Anxiety and OCD (pp. 113-142). Elsevier. https://doi.org/10.1016/B978-0-12-815915$6.00006-8$

Goday, P. S., Huh, S. Y., Silverman, A., Lukens, C. T., Dodrill, P., Cohen, S. S., Delaney, A. L., Feuling, M. B., Noel, R. J., Gisel, E., Kenzer, A., Kessler, D. B., Kraus de Camargo, O., Browne, J., \& Phalen, J. A. (2019). Pediatric Feeding Disorder: Consensus Definition and Conceptual Framework. Journal of Pediatric Gastroenterology and Nutrition, 68(1), 124129. https://doi.org/10.1097/MPG.0000000000002188

Greer, A. J., Gulotta, C. S., Masler, E. A., \& Laud, R. B. (2008). Caregiver Stress and Outcomes of Children with Pediatric Feeding Disorders Treated in an Intensive Interdisciplinary Program. Journal of Pediatric Psychology, 33(6), 612-620. https://doi.org/10.1093/jpepsy/jsm116

Kovacic, K., Rein, L. E., Szabo, A., Kommareddy, S., Bhagavatula, P., \& Goday, P. S. (2021). Pediatric Feeding Disorder: A Nationwide Prevalence Study. The Journal of Pediatrics, 228, 126-131.e3. https://doi.org/10.1016/j.jpeds.2020.07.047 
Laud, R. B., Girolami, P. A., Boscoe, J. H., \& Gulotta, C. S. (2009). Treatment Outcomes for Severe Feeding Problems in Children With Autism Spectrum Disorder. Behavior Modification, 33(5), 520-536. https://doi.org/10.1177/0145445509346729

Ledford, J. R., \& Gast, D. L. (2006). Feeding Problems in Children With Autism Spectrum Disorders: A Review. Focus on Autism and Other Developmental Disabilities, 21(3), 153166. https://doi.org/10.1177/10883576060210030401

Maenner, M. J. (2020). Prevalence of Autism Spectrum Disorder Among Children Aged 8 Years-Autism and Developmental Disabilities Monitoring Network, 11 Sites, United States, 2016. MMWR. Surveillance Summaries, 69. https://doi.org/10.15585/mmwr.ss6904a1

Marshall, J., Hill, R. J., Ziviani, J., \& Dodrill, P. (2014). Features of feeding difficulty in children with Autism Spectrum Disorder. International Journal of Speech Language Pathology, 16(2), 151-158. https://doi.org/10.3109/17549507.2013.808700

Mayes, S. D., \& Zickgraf, H. (2019). Atypical eating behaviors in children and adolescents with autism, ADHD, other disorders, and typical development. Research in Autism Spectrum Disorders, 64, 76-83. https://doi.org/10.1016/j.rasd.2019.04.002

Morris, S. E. (1989). Development of oral-motor skills in the neurologically impaired child receiving non-oral feedings. Dysphagia, 3(3), 135-154. https://doi.org/10.1007/BF02407132

Murat Baldwin, M., Xiao, Z., \& Murray, A. (2021). Temporal Synchrony in Autism: A Systematic Review. Review Journal of Autism and Developmental Disorders. https://doi.org/10.1007/s40489-021-00276-5 
Schafer, S. T., Paquola, A. C. M., Stern, S., Gosselin, D., Ku, M., Pena, M., Kuret, T. J. M., Liyanage, M., Mansour, A. A., Jaeger, B. N., Marchetto, M. C., Glass, C. K., Mertens, J., \& Gage, F. H. (2019). Pathological priming causes developmental gene network heterochronicity in autistic subject-derived neurons. Nature Neuroscience, 22(2), 243-255. https://doi.org/10.1038/s41593-018-0295-x

Schmitt, L., Heiss, C. J., \& Campbell, E. E. (2008). A Comparison of Nutrient Intake and Eating Behaviors of Boys With and Without Autism. Topics in Clinical Nutrition, 23(1), 23-31. https://doi.org/10.1097/01.TIN.0000312077.45953.6c

Schreck, K. A., Williams, K., \& Smith, A. F. (2004). A Comparison of Eating Behaviors Between Children with and Without Autism. Journal of Autism and Developmental Disorders, 34(4), 433-438. https://doi.org/10.1023/B:JADD.0000037419.78531.86

Seiverling, L. J., Hendy, H. M., \& Williams, K. E. (2011). Child and Parent Variables Associated with Texture Problems in Children's Feeding. Journal of Developmental and Physical Disabilities, 23(4), 303-311. https://doi.org/10.1007/s10882-011-9229-1

Sharp, W. G., Jaquess, D. L., Morton, J. F., \& Herzinger, C. V. (2010). Pediatric Feeding Disorders: A Quantitative Synthesis of Treatment Outcomes. Clinical Child and Family Psychology Review, 13(4), 348-365. https://doi.org/10.1007/s10567-010-0079-7

Sharp, W. G., Volkert, V. M., Scahill, L., McCracken, C. E., \& McElhanon, B. (2017). A Systematic Review and Meta-Analysis of Intensive Multidisciplinary Intervention for Pediatric Feeding Disorders: How Standard Is the Standard of Care? The Journal of Pediatrics, 181, 116-124.e4. https://doi.org/10.1016/j.jpeds.2016.10.002

Tarbox, J. (2017). Treating feeding challenges in autism: Turning the tables on mealtime. Academic Press. 
Tarbox, J., Schiff, A., \& Najdowski, A. C. (2010). Parent-Implemented Procedural Modification of Escape Extinction in the Treatment of Food Selectivity in a Young Child with Autism. Education and Treatment of Children, 33(2), 223-234. https://doi.org/10.1353/etc.0.0089

Taylor, T., Kozlowski, A. M., \& Girolami, P. A. (2017). Comparing behavioral treatment of feeding difficulties and tube dependence in children with cerebral palsy and autism spectrum disorder. NeuroRehabilitation (Reading, Mass.), 41(2), 395-402. https://doi.org/10.3233/NRE-162071

Volkert, V. M., Peterson, K. M., Zeleny, J. R., \& Piazza, C. C. (2014). A Clinical Protocol to Increase Chewing and Assess Mastication in Children With Feeding Disorders. Behavior Modification, 38(5), 705-729. https://doi.org/10.1177/0145445514536575

Volkert, V. M., Piazza, C. C., Vaz, P. C. M., \& Frese, J. (2013). A Pilot Study to Increase Chewing in Children With Feeding Disorders. Behavior Modification, 37(3), 391-408. https://doi.org/10.1177/0145445512474295

Williams, K. E., Riegel, K., Gibbons, B., \& Field, D. G. (2007). Intensive Behavioral Treatment for Severe Feeding Problems: A Cost-effective Alternative to Tube Feeding? Journal of Developmental and Physical Disabilities, 19(3), 227-235. https://doi.org/10.1007/s10882007-9051-y

World Health Organization. (2001). International classification of functioning, disability and health: ICF. World Health Organization. 